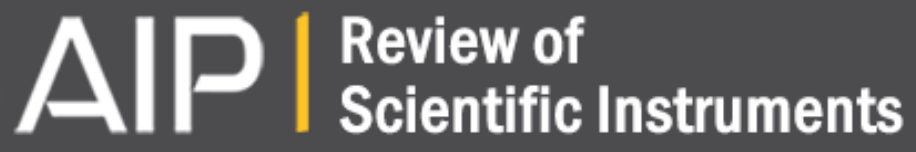

Diagnosing collisions of magnetized, high energy density plasma flows using a combination of collective Thomson scattering, Faraday rotation, and interferometry (invited)a)

G. F. Swadling, S. V. Lebedev, G. N. Hall, S. Patankar, N. H. Stewart, R. A. Smith, A. J. Harvey-Thompson, G.

C. Burdiak, P. de Grouchy, J. Skidmore, L. Suttle, F. Suzuki-Vidal, S. N. Bland, K. H. Kwek, L. Pickworth, M.

Bennett, J. D. Hare, W. Rozmus, and J. Yuan

Citation: Review of Scientific Instruments 85, 11E502 (2014); doi: 10.1063/1.4890564

View online: http://dx.doi.org/10.1063/1.4890564

View Table of Contents: http://scitation.aip.org/content/aip/journal/rsi/85/11?ver=pdfcov

Published by the AIP Publishing

Articles you may be interested in

High-frequency fluctuation measurements by far-infrared laser Faraday-effect polarimetry-interferometry and forward scattering system on MSTa)

Rev. Sci. Instrum. 85, 11D406 (2014); 10.1063/1.4889909

The formation of reverse shocks in magnetized high energy density supersonic plasma flowsa)

Phys. Plasmas 21, 056305 (2014); 10.1063/1.4874334

Magnetic field measurements in low density plasmas using paramagnetic Faraday rotator glassa)

Rev. Sci. Instrum. 83, 10D503 (2012); 10.1063/1.4728214

Differential interferometry for measurement of density fluctuations and fluctuation-induced transport (invited)a)

Rev. Sci. Instrum. 81, 10 D509 (2010); 10.1063/1.3464480

C O 2 laser polarimeter for Faraday rotation measurements in the DIII-D tokamaka)

Rev. Sci. Instrum. 79, 10E719 (2008); 10.1063/1.2955859
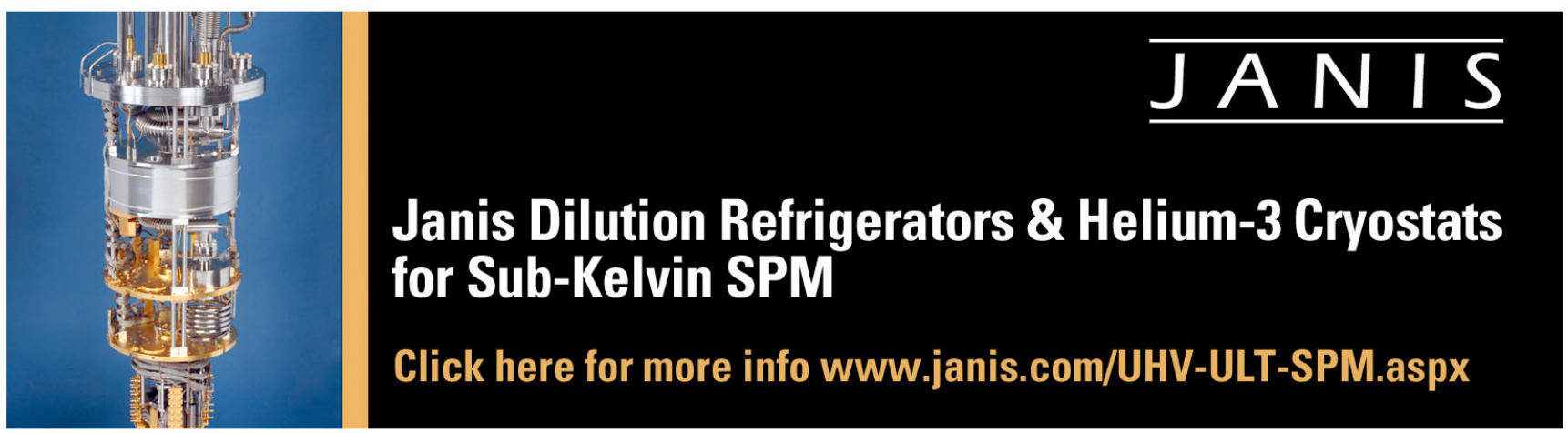


\title{
Diagnosing collisions of magnetized, high energy density plasma flows using a combination of collective Thomson scattering, Faraday rotation, and interferometry (invited) $)^{\text {a) }}$
}

\author{
G. F. Swadling, ${ }^{1, \text { b) }}$ S. V. Lebedev, ${ }^{1}$ G. N. Hall, ${ }^{1, c)}$ S. Patankar, ${ }^{1}$ N. H. Stewart, ${ }^{1}$ \\ R. A. Smith, ${ }^{1}$ A. J. Harvey-Thompson, ${ }^{2}$ G. C. Burdiak, ${ }^{1}$ P. de Grouchy, ${ }^{1, d)}$ J. Skidmore, ${ }^{1, e)}$ \\ L. Suttle, ${ }^{1}$ F. Suzuki-Vidal, ${ }^{1}$ S. N. Bland, ${ }^{1}$ K. H. Kwek, ${ }^{1}$ L. Pickworth, ${ }^{1, c)}$ M. Bennett, ${ }^{1}$ \\ J. D. Hare, ${ }^{1}$ W. Rozmus, ${ }^{3}$ and J. Yuan ${ }^{4}$ \\ ${ }^{1}$ Plasma Physics Group, Imperial College, London SW6 7LZ, United Kingdom \\ ${ }^{2}$ Sandia National Laboratory, Albuquerque, New Mexico 87185-1193, USA \\ ${ }^{3}$ Department of Physics, University of Alberta, Edmonton, Alberta T6G 2J1, Canada \\ ${ }^{4}$ Key Laboratory of Pulsed Power, Institute of Fluid Physics, CAE, Mianyang 621900, China
}

(Presented 3 June 2014; received 22 May 2014; accepted 25 June 2014; published online 28 July 2014)

A suite of laser based diagnostics is used to study interactions of magnetised, supersonic, radiatively cooled plasma flows produced using the Magpie pulse power generator (1.4 MA, $240 \mathrm{~ns}$ rise time). Collective optical Thomson scattering measures the time-resolved local flow velocity and temperature across 7-14 spatial positions. The scattering spectrum is recorded from multiple directions, allowing more accurate reconstruction of the flow velocity vectors. The areal electron density is measured using 2D interferometry; optimisation and analysis are discussed. The Faraday rotation diagnostic, operating at $1053 \mathrm{~nm}$, measures the magnetic field distribution in the plasma. Measurements obtained simultaneously by these diagnostics are used to constrain analysis, increasing the accuracy of interpretation. @ 2014 AIP Publishing LLC. [http://dx.doi.org/10.1063/1.4890564]

\section{INTRODUCTION}

The collisions of plasma flows and the dynamics of the resulting interactions are a topic of great interest to the plasma physics community. These phenomena are ubiquitous in astrophysical plasmas, and have formed the focus of a number of recent high energy density physics (HEDP) and laboratory astrophysics experiments. ${ }^{1-4}$ This paper presents a suite of laser-based diagnostics that have been developed in order to study the interactions of high temperature, supersonic, magnetized, high atomic number plasma flows. These flows are produced using the Magpie ${ }^{5}$ pulsed power generator at Imperial College (1.4 MA, $240 \mathrm{~ns})$; the flows are accelerated by the $J \times B$ forces that arise due to the interaction of the current conducted by the plasma with the associated selfmagnetic field. Careful design of the load allows the geometry of the flows to be controlled and focused in order to produce quasi-1D flows, ${ }^{6,7}$ cylindrically symmetric interactions, ${ }^{8}$ or jet into ambient medium interactions. ${ }^{9,10}$ The collisional scale lengths of the interactions can be tuned both through the choice of the element used to produce the plasmas (typically

\footnotetext{
a) Invited paper, published as part of the Proceedings of the 20th Topical Conference on High-Temperature Plasma Diagnostics, Atlanta, Georgia, USA, June 2014.

b) Author to whom correspondence should be addressed. Electronic mail: george.swadling@imperial.ac.uk

c) Present address: Lawrence Livermore National Laboratory, 7000 East Avenue, Livermore, California 94550, USA.

d) Present address: Laboratory of Plasma Studies, Cornell University, 439 Rhodes Hall, Ithaca, New York 14853, USA

e) Present address: AWE, Aldermaston, Reading, Berkshire RG74PR, United Kingdom.
}

tungsten or aluminium) and through control of the timing of measurements, allowing the study of both shock formation and long-range flow interpenetration. The current-driven acceleration mechanism used in these experiments means that the plasma flows are magnetised at the point of launch and estimates of the relevant magnetic Reynolds numbers indicate that in many cases this field is advected along with the flows into the interaction region. ${ }^{11}$ This provides the opportunity to study the dynamics of magnetised flow interactions and magnetised shock formation.

Three separate laser-driven diagnostic systems have been developed in order to study the dynamics of interacting flows in greater detail. These diagnostics are designed to measure the evolution of the plasma parameters as the flows interact. The optical Thomson scattering (TS) diagnostic was developed chiefly to study the variations in flow velocity $v$ of the plasma, by measuring the Doppler shift of the collectiveregime ion-feature. Scattering is collected from multiple angles, allowing the system to measure multiple orthogonal components of the overall $v$ vector. Fitting the spectral shape of the ion-feature also allows measurement of the ion temperature $T_{i}$, and in some cases the product of the average ionisation state and electron temperature, $\bar{Z} T_{e}$. The diagnostic was initially used to study ablation flows in wire array zpinches ${ }^{12,13}$ and more recently to study plasma flow interpenetration and interaction on the axis of an array. ${ }^{11}$ A Faraday polarimetry diagnostic has been developed in order to make temporally and spatially resolved measurements of the magnetic field in the plasma. This diagnostic is being applied to study the transport of magnetic fields by the plasma flows and the effect of these transported fields on the dynamics of 
the flow interactions. Proper analysis of both the TS and the Faraday data requires a simultaneous measurement of the plasma electron density. This is achieved using laser interferometric imaging. The interferometry diagnostic has been used extensively in previous Magpie experiments to study the interactions of wire array ablation flows, ${ }^{6,8}$ the interactions of flows with solid targets, ${ }^{7}$ and the interactions of jets with ambient media. ${ }^{10,14}$ Combining the information acquired through these different diagnostic techniques allows additional constraints to be applied during analysis of experimental data, which in turn leads to a more accurate interpretation of the results.

\section{THOMSON SCATTERING}

The Magpie TS diagnostic records the spectral profile of the TS ion-feature, the part of the scattered spectrum produced by collective, coherent scattering of the probe laser by the clouds of electrons that screen the charge of each ion. The spectral shape of the ion-feature is sensitive to $v, T_{i}$ (the ion temperature), and $\bar{Z} T_{e}$ (the product of the average ionisation state of the ions and the electron temperature). Analysis involves fitting the recorded spectral profiles using the nonrelativistic, Maxwellian spectral density function $S(\omega, \boldsymbol{k}){ }^{15}$ An important feature of the TS setup used on Magpie is that it can simultaneously measure the scattered spectrum from multiple angles, allowing independent measurements of the orthogonal components of $\boldsymbol{v}$. Combining these measurements results in a more accurate reconstruction of the full $\boldsymbol{v}$ vector. A vector diagram of one of the scattering geometries used in experiments is shown in Figure 1(a). The spectrum of scattering observed from any given direction is sensitive only to the component of $v$ which lies parallel to the specific scattering vector $\boldsymbol{k}_{\boldsymbol{s}} \equiv \boldsymbol{k}_{\boldsymbol{o}}-\boldsymbol{k}_{\boldsymbol{i}}$. The wavevector of the incoming probe, $\boldsymbol{k}_{\boldsymbol{i}}$, is fixed, and the direction of the observed wavevector $\left(\hat{\boldsymbol{k}}_{\boldsymbol{o}}\right)$ is constant. As the range of variation in the magnitude of the observed wavevector $\left|\boldsymbol{k}_{\boldsymbol{o}}\right|$ is very small over the spectrometer's sensitivity range, the measured component of $v$ may be treated as approximately constant for observations in any particular direction. The collection directions $\hat{\boldsymbol{k}}_{\boldsymbol{o}}$ are selected such that the resulting $\boldsymbol{k}_{\boldsymbol{s}}$ lie parallel to the two desired orthogonal components of $\boldsymbol{v}$. The ion velocity distribution function $f_{i}$, and therefore $T_{i}$ is also measured along these two orthogonal Cartesian axes; this can be used to distinguish between situations where $T_{i}$ represents a truly isotropic temperature and cases where an enhanced "effective" ion temperature is measured due to the interpenetration of multiple ion flows traveling in different directions in a single plane.

Figure 1(b) shows a diagram of the Thomson scattering diagnostic setup that corresponds to the scattering geometry shown in (a). The probe laser beam (8 ns, $3 \mathrm{~J}, 532 \mathrm{~nm}$ ) is focused through the experiment and undergoes scattering by the plasma. Lenses collect and image scattered light from two different directions onto the inputs of a pair of linear fibre optic arrays (manufactured by LEONI), each consisting of either 7 $\times 200 \mu \mathrm{m} \varnothing$ fibres, spaced by $390 \mu \mathrm{m}$ (overall array length of $2.34 \mathrm{~mm}$ ), or $14 \times 100 \mu \mathrm{m} \varnothing$ fibres, spaced by $250 \mu \mathrm{m}$, with an overall array length of $3.25 \mathrm{~mm}$. The fibres couple the scattered light to a $0.5 \mathrm{~m}, 2400$ lines $/ \mathrm{mm}$ imaging spectrograph
(ANDOR Shamrock 500), and the spectrum is recorded using a time gated (4 ns) intensified charge coupled device (ICCD) camera (ANDOR iStar). The time gate for the camera is triggered using a photodiode which monitors backscatter from one of the probe beam delivery optics; this method of triggering minimises timing jitter, which in turn minimises the exposure time required in order to guarantee the scattered signal is observed. This is important as the noise introduced by self-emission from the plasma is integrated over the exposure time.

The overall spectral resolution achieved depends on the diameter of the fibres used, $0.25 \AA$ for $100 \mu \mathrm{m}, \sim 0.5 \AA$ for $200 \mu \mathrm{m}$; although the resolution is improved when using $100 \mu \mathrm{m} \emptyset$ fibres, the total amount of scattered light collected per fibre is reduced. The arrays of fibres are aligned along the lengths of the images of the probe beam, such that each fibre collects light from a volume of plasma at a different spatial position. The positions of these volumes are spaced equally along the probe beam, with the spacing and spatial resolution in the direction parallel to the probe beam set by the magnification of the imaging system and the relative angles between the incoming probe vector and the collection vectors. The spatial resolution perpendicular to the probe beam is set by the diameter of the probe beam. The probing laser beam has an initial collimated diameter of $\sim 2.5 \mathrm{~cm}$ and is focused through the centre of the plasma by an $f=2.5 \mathrm{~m}$ lens. Assuming the beam has a perfect Gaussian beam profile, the diffraction-limited beam waist at focus is $w_{0} \sim 70 \mu \mathrm{m}$, with a Rayleigh length of $z_{R} \sim 13 \mathrm{~mm} .{ }^{16}$ Imperfections in the beam profile mean the actual focal spot size is likely larger, however it is unlikely that the beam diameter exceeds $300 \mu \mathrm{m}$ at any point over a range $\pm 13 \mathrm{~mm}$ either side of focus; this may therefore be treated as an upper limit for the transverse spatial scale of the scattering volumes. Alignment of the probe beam and collection fibres is performed under vacuum using a fine metal pin $(100 \mu \mathrm{m} \varnothing)$ mounted on a micrometer-driven adjustable kinematic stage. The position of the pin is adjusted so that it passes through the desired scattering volume, the probe beam alignment is adjusted to maximise scattering off of the pin, and finally the light scattered by the pin is used to align one of the fibres to maximise collection. Often it is desirable to collect scattered radiation simultaneously from the same volumes along both collection vectors; this alignment method guarantees that in such cases both fibre arrays collect light from exactly the same set of volumes.

An example of the raw data produced by the TS diagnostic is shown in Figure 1(c); TS is used here to diagnose the behaviour of interpenetrating, radially convergent tungsten ablation streams in the region around the axis of an 8-wire cylindrical wire array z-pinch during the early phase of the current drive $(\sim 120 \mathrm{~ns})$, when the mean free path for the streams is still significant compared to the experimental length scale. ${ }^{8,11}$ Figure 1(d) shows an electron density $\left(n_{e}\right)$ map extracted from an end-on interferogram, captured along the path indicated in (b). The two arrays of fibers collected scattered light from the same set of seven scattering volumes, distributed evenly within $r=1.5 \mathrm{~mm}$ either side of the axis; these positions are marked on (d) with red circles. Collection vectors were selected in order to independently measure the axial $\left(v_{z}\right)$ 


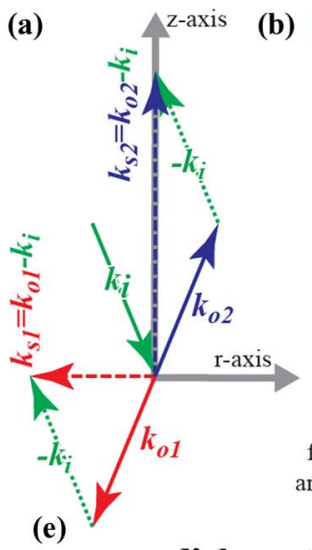

(e)

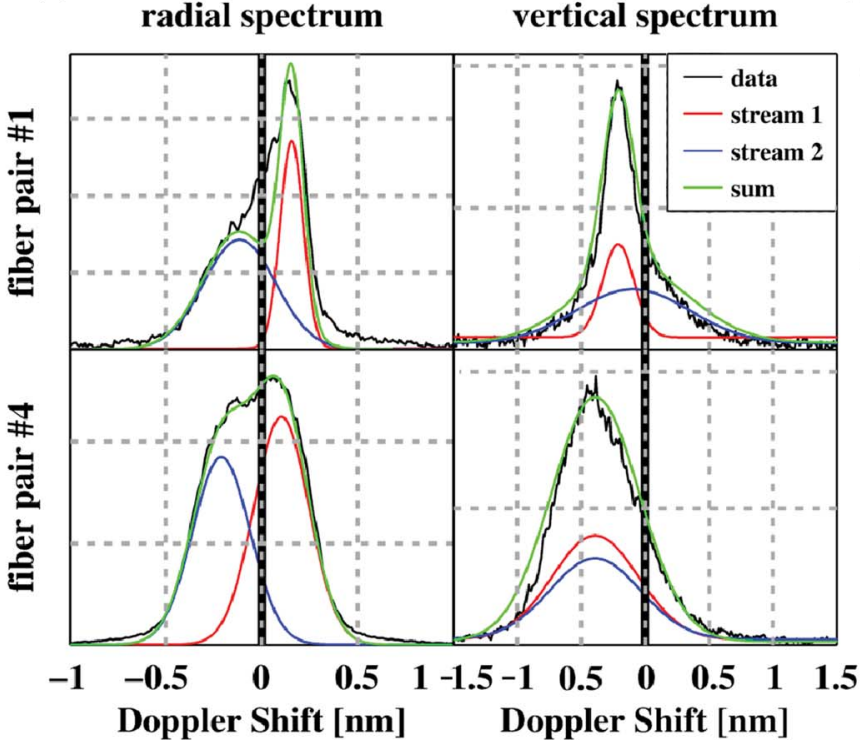

(c)

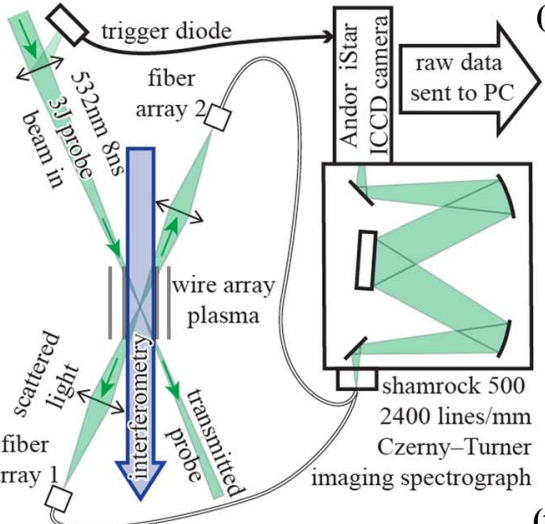

2

(f)

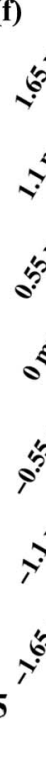

(f)
Raw Spectrogram

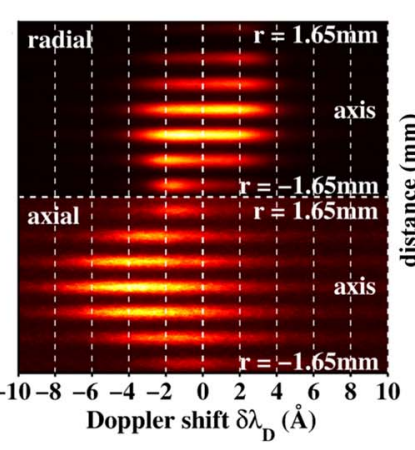

(d) Analysed Interferometry data with thomson scattering volumes

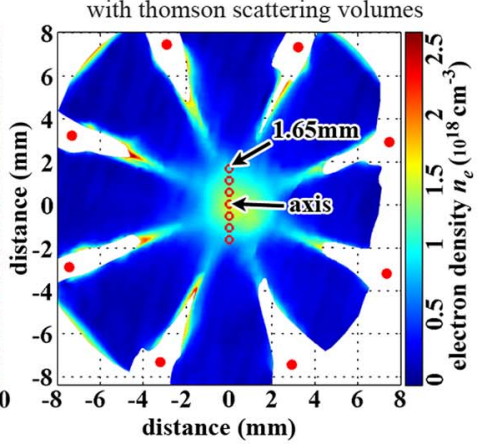

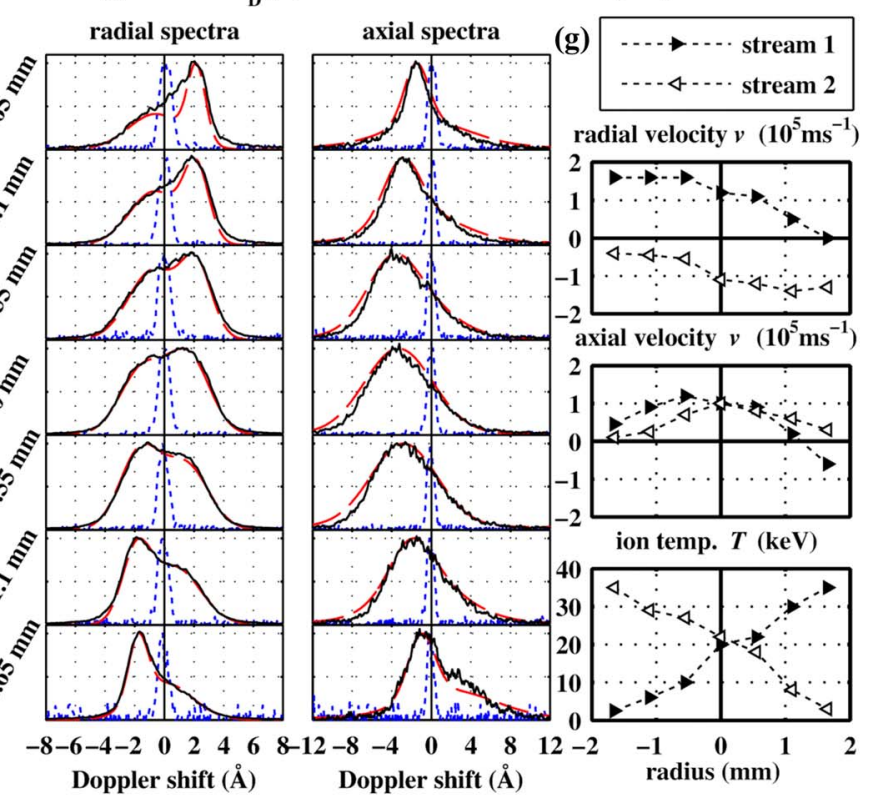

FIG. 1. (a) Thomson Scattering (TS) geometry, (b) experimental setup, (c) raw TS data, (d) $n_{e}$ map with scattering volumes (red circles) and wire positions (red dots), (e) construction of spectral fits for interpenetrating flows, (f) constrained spectral fits for all seven volumes, (g) variation in plasma parameters used to construct fits. Parts (c), (d), (f), and (g) reproduced with permission from G. F. Swadling et al., Phys. Rev. Lett. 113, 035003 (2014). Copyright 2014 American Physical Society. ${ }^{11}$

and radial $\left(v_{r}\right)$ velocity components (see (a)). Fittings for the spectra were calculated using the multiple ion species equation for $S\left(\omega, \boldsymbol{k}_{s}\right) .{ }^{15}$ Each fit consists approximately of a sum of component spectra from the two interpenetrating flows. Spectra taken from the same scattering volumes, but observed from different scattering vectors, were fitted simultaneously; they share the same $T_{e}$ and $\bar{Z}$ but have different $v, T_{i}$, and $n_{i}$. The total $n_{e}$, measured by interferometry, was used as a further fitting constraint. The construction of the fits is illustrated in Figure 1(e) for two of the seven pairs of fibers. We see that the two components have different widths and heights, but the total spectra appear to be good matches for the observed spectrum. Figure 1(f) shows the final fits for the radial and axial spectra; the variations in the plasma conditions used to calculate these fits are plotted in Figure 1(g), and illustrate how the two ablation streams decelerate as they approach the axis and interpenetrate. The two streams are also deflected axially, both in the same direction, towards the anode of the generator. This deflection suggests the presence of a toroidal magnetic field on axis, likely brought to the axis by the ablation flows. ${ }^{11}$ The data also show the temperatures of the two streams increases fairly linearly from $\sim 2 \mathrm{keV}$ to $\sim 20 \mathrm{keV}$ as they pass through the axis, and peaking at $\sim 35 \mathrm{keV}$. The independent axial and radial measurements indicate, as discussed earlier, that this is a true isotropic $T_{i}$, rather than an "effective" $T_{i}$.

\section{LASER INTERFEROMETRIC IMAGING}

The laser interferometric imaging (interferometry) diagnostics on Magpie are fielded in a wide variety of experiments. Interferometry is directly sensitive to the path integrated (areal) free electron density $\left(\int n_{e} d l\right)$ of the plasma, and thus provides a means of making quantitative measurements of the plasma parameters in the experiment. Previous interferometry diagnostics fielded on Magpie have probed the plasma with 2nd $(532 \mathrm{~nm})$ and 3rd $(355 \mathrm{~nm})$ harmonic ND-YAG laser pulses, provided by of an EKSPLA SL321P $(500 \mathrm{ps}, 500 \mathrm{~mJ})$. The temporal resolution of the resulting interferograms is set by the laser pulse duration; the CCDs used to capture the images (Canon 350D and 450D DSLRs) are left exposed for the entire duration of the experiment. A delay may be introduced between the two beams in order to allow multiple probing times along the same line during each 


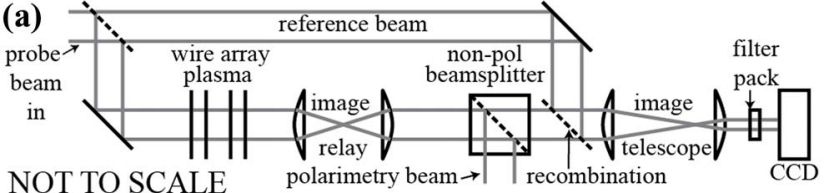

(b) Experiment cross section (c) Analysed data
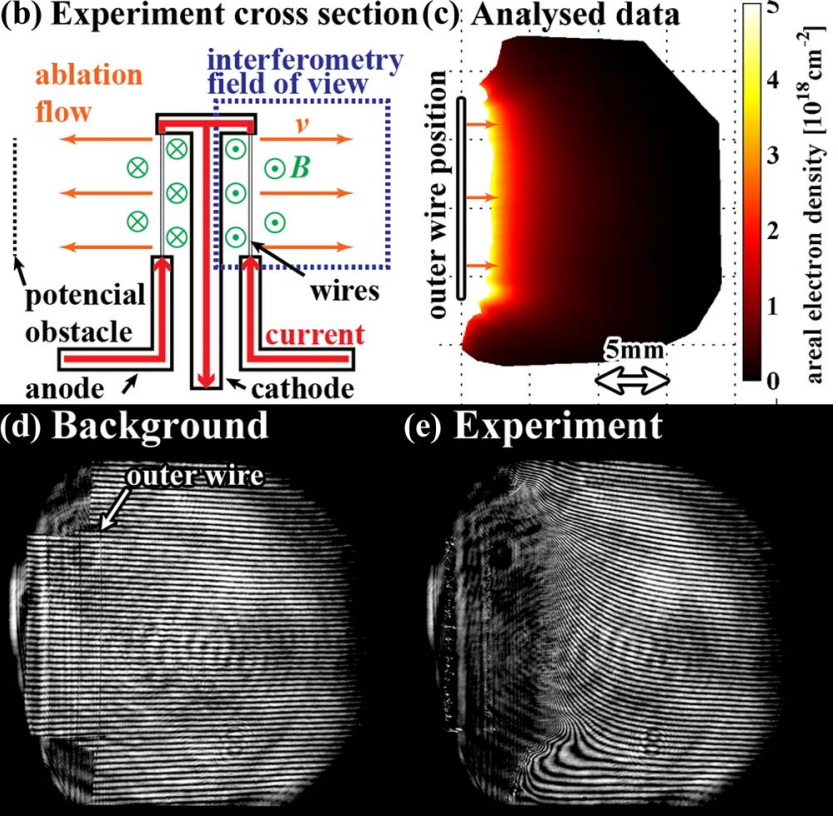

FIG. 2. (a) Schematic of Mach-Zehnder interferometric imaging diagnostic, (b) experimental layout, (c) analysed areal electron density map, (d) background interferogram, (e) experimental interferogram.

experiment, and the probes beams may also be split to allow probing from multiple angles simultaneously. The interferometers use a Mach-Zehnder design (see Figure 2(a)) as the large size of the plasmas measured preclude many of the other options. Although these interferometers can be difficult to align, the flat reference beams they provide do lead to a number of advantages in terms of simplicity of interpretation and analysis.

The relatively large spatial scales $(\sim 1 \mathrm{~cm})$ and high electron densities $\left(10^{19} \mathrm{~cm}^{-3}\right)$ of the plasmas studied in Magpie experiments mean that interferogram are often characterised by large and contorted fringe shifts. ${ }^{6}$ The recorded intensity of time-integrated self-emission from the plasma can be significant, and fringe contrast can be reduced or lost in regions where density gradients are strong enough to deflect the probe beam outside of the optical collection angle $\left(\theta_{\alpha}\right)$ of the imaging system. Finally the finite depth of focus of the imaging optics combined with the large physical extent of the objects can introduce extra, unwanted effects over the top of the fringe patterns. The combination of these effects means in many cases analysis using standard or automated methods such as Fourier filtering is difficult. Custom tools have been developed ${ }^{6}$ in order to extract accurate phase maps from these noisy interferograms; the position of each of the fringes is traced using a combination of automatic and manual techniques, and then each is assigned a relative fringe number. The result is effectively a contour map of the interference phase. The "phase contours" are interpolated using a contour-optimised triangulation and in- terpolation technique, based on similar methods used in topographical reconstruction; ${ }^{17}$ this approach avoids problems associated with other scattered data interpolation methods, such as Delaunay triangulation, which can introduce so called "flat feature" artefacts when used to interpolate contour-like datasets. The improved triangulation effectively "stitches" together adjacent contours, resulting in a smoother and more accurate interpolated phase map. Tracing and interpolation are carried out for both the background and experimental interferograms. The phase change induced by the plasma is then calculated by subtracting the two interpolated phase maps, and this is calibrated using the free electron refractive index to produce maps of $\int n_{e} d l$.

Even with the introduction of improved analysis techniques, one of the main limitations of the interferometry diagnostics fielded on Magpie previously is the typical laser energy available to probe the plasma. At the point at which it reaches the plasma, the probe beam energy is typically $\sim 100 \mathrm{~mJ}$, with a diameter of $10-50 \mathrm{~mm}$, depending on the experiment. Even using narrow band pass filters $(\delta \lambda \sim 3 \mathrm{~nm})$, interference patterns are often obscured by time-integrated self-emission from the plasma. To reduce the amount of collected self-emission apertures are placed at the first focus of the imaging system. These typically limit the acceptance angle of the camera to $\theta_{\alpha}<0.005 \mathrm{rad}$; while this reduces the solid angle of self-emission collected, it also places a limit on the strength of the transverse $\int n_{e} d l$ gradients that the diagnostic is capable of probing. This limitation can be a particular problem when studying strong shocks. The newly developed Faraday diagnostic requires an interferometry channel in order to calibrate measurements of the magnetic field. The probe for this diagnostic is provided by a long pulse arm of the new Cerberus ND-Glass laser system $(\sim 10 \mathrm{~J}, \lambda=1053 \mathrm{~nm}$, $1 \mathrm{~ns}$ ); probing is carried out using the fundamental laser wavelength in order to maximise sensitivity to the Faraday effect (scales as $\lambda^{2}$ ), however this choice exacerbates the problems discussed above. Interferometers measure the phase shift induced in the probe beam via the fringe shift $\left(f_{s}\right)$. In cases where the electron density $n_{\mathrm{e}}$ of the plasma is much lower than the critical density $n_{c}(\lambda), f_{s}$ is directly proportional to $\int n_{e} d l:^{18}$

$f_{s} \sim \frac{-\pi}{\lambda n_{c}} \int n_{e} d l=-\frac{r_{e} \lambda}{2 \pi} \int n_{e} d l ; \quad r_{e}=2.82 \times 10^{-15} \mathrm{~m}[S I]$.

The sensitivity of the interferometer thus scales with $\lambda$, and $1053 \mathrm{~nm}$ probing is therefore approximately twice as sensitive as $532 \mathrm{~nm}$ probing. Unfortunately, the angle the probe is deflected $(\theta)$ by transverse gradients in $\int n_{e} d l$ scales with $\lambda^{2}$ :

$$
\theta \sim \lambda \frac{d f_{s}}{d r} \sim-\frac{r_{e} \lambda^{2}}{2 \pi} \frac{d}{d r} \int n_{e} d l .
$$

Thus the $1053 \mathrm{~nm}$ system requires $\sim 4$ times greater $\theta_{\alpha}$ than the equivalent $532 \mathrm{~nm}$ system to successfully probe the same density gradient. As the total energy of the $1053 \mathrm{~nm}$ beam is $\sim 100$ times that of the $532 \mathrm{~nm}$ beam used previously, the $\theta_{\alpha}$ of the collection optics may potentially be increased by up to a factor of $\sim 10$, whilst still maintaining a similar signal to noise ratio. The $1053 \mathrm{~nm}$ imaging system therefore has the potential to image density gradients up to $\sim 2.5$ times stronger 
than the previous system. In practice $\theta_{\alpha}$, and therefore maximum gradient resolvable is currently limited by the size of the available optics.

As an example, Figure 2 contains $1053 \mathrm{~nm}$ interferometry data captured during an experiment carried out to measure the $\boldsymbol{B}$ field embedded in an outflow of plasma produced by an inverse cylindrical wire array $;^{7,19,20}$ (b) shows a sideon schematic view of this load configuration, (c) shows the $\int n_{e} d l$ map that results from analysis of interferograms (d) and (e) using the methods described above. The inverse wire array produces ablation streams which are accelerated radially away from the array axis due to the reversal of the standard wire array z-pinch magnetic field topology. This setup is interesting as it allows the study of the interaction of magnetised flows with external obstacles. ${ }^{7}$ These data demonstrate the dynamic range of sensitivity achieved by this interferometer; the peak density in this plot, $\sim 5 \times 10^{18} \mathrm{~cm}^{-2}$ corresponds to $\sim 20$ fringe excursions, while the accuracy of the analysis method is conservatively estimated at $\sim 1 / 5$ of a fringe shift $\left(\sim 4 \times 10^{16} \mathrm{~cm}^{-3}\right)$. This corresponds to a dynamic range of $\sim 100$. The peak gradient in the fringe shift, measured at the point where fringe contrast is just lost, is $\sim 10$ fringes $/ \mathrm{mm}$. This corresponds to $\theta_{\alpha} \sim 0.01 \mathrm{rad}\left(\sim 0.6^{\circ}\right)$, double that currently achieved by the other Magpie interferometers. Further increases in $\theta_{\alpha}$ should allow the system to image even deeper into strong density gradients. This electron density data will be combined with the polarimetry measurements of the Faraday effect presented in Sec. IV, in order to determine the magnetic field in the flow.

\section{FARADAY ROTATION IMAGING}

Faraday rotation imaging is used to make spatially and temporally resolved, non-disruptive measurements of the line-averaged magnetic field $\left(\boldsymbol{B}_{\|}\right)$embedded in the plasma, through the measurement of the angle of rotation $(\alpha)$ induced in a plane polarised probe laser beam. This technique has a number of advantages over other commonly used methods of measuring the magnetic field $(\boldsymbol{B})$ in a plasma, such as placing "B-dot" magnetic pickup coils ${ }^{21-24}$ in the path of the plasma flow. These probes can cause significant disruption to the flow and the signals they produce are subject to noise associated both with coupling of the drive voltage to the straycapacitance of the coil and with shorting/shielding of the coil by the plasma. In contrast, Faraday rotation laser probing does not disturb the plasma and is insensitive to the drive electric field. The spatial resolution of B-dot probes is limited to their cross-sectional area, and each one can only measure $\boldsymbol{B}$ in one spatial position, making measurements of the overall $\boldsymbol{B}$ field structure difficult; the spatial resolution of Faraday measurements is limited only by the spatial resolution of the optical setup and detectors used, and the $\boldsymbol{B}$ field can be measured over the entire cross-section of the probe beam, meaning that the full line-integrated, 2-D field structure can be observed with a single measurement.

Faraday imaging is not a novel diagnostic approach in pulsed power driven HEDP experiments; similar diagnostics have been developed in order to study the dynamics of fibre implosions, ${ }^{25}$ wire array z-pinches and X-pinches,${ }^{26-28}$ gas puffs, ${ }^{29}$ and plasma foci, ${ }^{30}$ however in those previous experiments probing was carried out using a $\lambda=532 \mathrm{~nm}$ laser (2nd Harmonic ND:YAG). At this wavelength the angles of rotation induced are typically very small $\left(\sim 1^{\circ}\right)$, and therefore difficult to measure accurately. In order to produce measurable contrast over such small rotations the apparatus must be operated very near the extinction angle of the polarisation analysers however this increases the effects of noise associated with the plasma's un-polarised self-emission. The Faraday rotation diagnostic developed for Magpie takes advantage of the $\lambda^{2}$ scaling of $\alpha$ by using a $\lambda=1053 \mathrm{~nm}$ probe to quadruple the effective sensitivity. The larger values of $\alpha$ produced mean that this diagnostic should be able to achieve much greater signal to noise ratios. In addition to this change, the probe beam has been made significantly more energetic than those used in previous experiments, $\sim 5 \mathrm{~J}$ compared to 25-300 $\mathrm{mJ}$ (energy loss here is due to splitting off of reference beam); this results in another significant improvement in the signal-to noise ratio, and means that the imaging system can be designed with a large acceptance angle, allowing the system to image deeper into the strong density gradients associated with the shocks fronts formed between colliding plasma flows.

In order to measure $\boldsymbol{B}, \alpha$ must first be measured using polarimetry. After passing through the experiment the probe beam is split into two channels, which are analysed at angles $\pm \beta$ either side of extinction. Four images (polarograms) are required in total; two from each channel, one (background) before and one during the experiment. The intensity distributions of the four polarograms are written

$$
\begin{aligned}
& I_{B \pm}(x, y)=s_{ \pm}(x, y) I_{B}(x, y) \sin ^{2}(\beta), \\
& I_{S \pm}=s_{ \pm}(x, y)\left[I_{s}(x, y) \sin ^{2}(\alpha(x, y) \pm \beta)+\frac{I_{S E}(x, y)}{2}\right] .
\end{aligned}
$$

Here the \pm shorthand has been used to write the equations for the pairs of $\pm \beta$ channels in single lines. $I_{B}(x, y)$ and $I_{S}(x, y)$ are the intensity distributions of the probe beams used to make the background and experimental (shot) measurements respectively, $I_{S E}(x, y)$ is the intensity distribution of the unpolarised plasma self-emission and $s_{-}(x, y), s_{+}(x, y)$ are the effective sensitivities of the two CCDs (taking into account any differences between polariser/filter/window transmissions). First the background polarograms are used to normalise the shot polarograms; this step removes any dependency on differences between the effective detector sensitivities:

$$
\frac{I_{S \pm}}{I_{B \pm}}=\frac{I_{S}}{I_{B}} \frac{\sin ^{2}(\alpha(x, y) \pm \beta)}{\sin ^{2}(\beta)}+\frac{I_{S E}(x, y)}{2 I_{B} \sin ^{2}(\beta)} .
$$

Taking the difference of these normalised intensity distributions removes the effects of self-emission:

$$
\begin{aligned}
D(x, y) & =\frac{I_{S+}}{I_{B+}}-\frac{I_{S-}}{I_{B-}}=\frac{I_{S}}{I_{B}} \frac{\sin ^{2}(\alpha+\beta)-\sin ^{2}(\alpha-\beta)}{\sin ^{2}(\beta)} \\
& =\frac{I_{S}}{I_{B}} \frac{2 \sin (2 \alpha)}{\tan \beta} .
\end{aligned}
$$


This expression is then rearranged to find $\alpha(x, y)$ as a function of the four polarograms:

$$
\begin{aligned}
\alpha(x, y)= & \frac{1}{2} \sin ^{-1}\left[\frac { I _ { B } ( x , y ) } { I _ { S } ( x , y ) } \left\{\frac{I_{S+}(x, y)}{I_{B+}(x, y)}\right.\right. \\
& \left.\left.-\frac{I_{S-}(x, y)}{I_{B-}(x, y)}\right\} \frac{\tan \beta}{2}\right] .
\end{aligned}
$$

The normalised spatial intensity distribution of the probing beam remains largely constant for the background and experimental polarograms; unavoidable spatial structure in the beam (e.g., diffraction rings from dust in the optical system) is normally constant across successive shots and is therefore normalised out by $I_{B}(x, y) / I_{S}(x, y)$. The above expression may therefore be further approximated by replacing $I_{B} / I_{S}$ with the ratio of the energy delivered by the background and experimental probe beams, which can be measured by a laser energy meter using a small percentage pick off of the full probe beam. The reproducibility of the Cerberus probe beam is good enough that so far in most cases this ratio has been $\sim 1$ to within a few percent.

The choice of analysing angle $\beta$ is very important, particularly when attempting to measure very small values of $\alpha$. The probe beam can be attenuated arbitrarily, however it is not possible to introduce an intensity offset. As a result the dynamic sensitivity range of the CCD will always lie between zero and some upper bound, and ideally the polarimetry measurement should use as much of this range as possible. The maximum achievable sensitivity of the polarimetry measurement over the desired measurement range $\alpha=\left[-\alpha_{\mathrm{M}}, \alpha_{M}\right]$ as a function of $\beta$ is given by the following expression (derivation in the Appendix):

$$
\left|\frac{d D\left(\alpha_{M}, \beta\right)}{d \alpha}\right|=\frac{\sin \left(2 \alpha_{M}\right) \sin (2 \beta)}{\alpha_{M} \sin ^{2}\left(\alpha_{M}+\beta\right)+\frac{I_{S E}}{2 I_{0}}},
$$

where the sensitivity is parameterised using the average value of $d D / d \alpha$; this is justified in cases where $\beta<30^{\circ}$, as the variation in $D$ is almost linear with $\alpha$ and therefore $d D / d \alpha$ is approximately constant across the range of interest.

Figure 3 contains plots of $|d D / d \alpha|$ for various different sets of measurement parameters. In cases where self-emission is negligible $\left(I_{S E} / I_{O}=0\right.$, solid lines in figure), the best choice of offset is $\beta=\alpha_{M}$ (vertical lines mark these peaks). For $\alpha_{\mathrm{M}}$ $<45^{\circ}$, this choice of $\beta$ results in the intensity of the two channels varying monotonically (in opposite directions) between zero and some upper limit over the range $\alpha=\left[-\alpha_{\mathrm{M}}, \alpha_{M}\right]$. Any rotation signal will unambiguously produce a brightening of the probe in one image and darkening in the other. If the beam is attenuated such that the background image intensity is $1 / 4$ of the CCD maximum, then the measured intensity variation will extend over the entire available dynamic range. Note that the sensitivity $(|d D / d \alpha|)$ achievable decreases inversely with increasing $\alpha_{M}$; this is because the same detection range must be spread over a broader range of possible angles. Figure 3 also illustrates how as the plasma self-emission increases, the optimum choice of $\beta$ also increases; for $\alpha_{M}=2^{\circ}$, for example, an increase in the self-emission ratio to $I_{S E} / I_{0}=0.01$, shifts the sensitivity peak to $\beta \sim 4^{\circ}$, almost double the optimum in the absence of self-emission

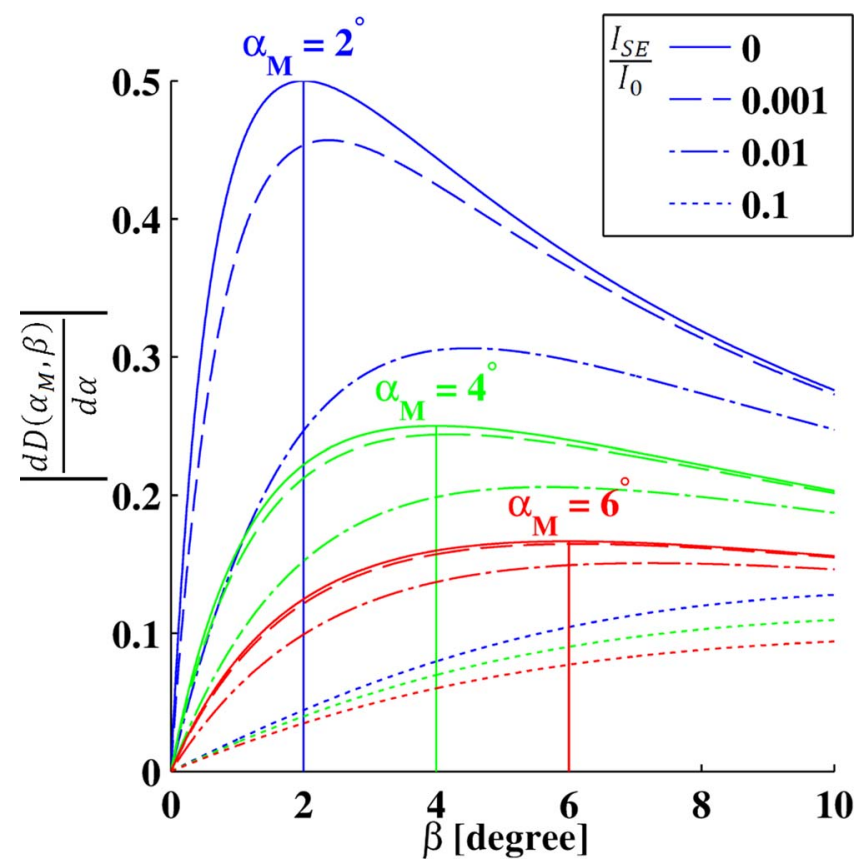

FIG. 3. Plots of the mean differential sensitivity achievable for measurement ranges $\alpha=\left[-\alpha_{\mathrm{M}}, \alpha_{M}\right]$ and self-emission ratios $\left(I_{S E} / I_{0}\right)$ for different choices of polariser bias angle $(\beta)$.

(the peak value of $|d D / d \alpha|$ also decreases by $\sim 40 \%$ ). The reason for this shift in the optimum value of $\beta$ is that while the effective intensity of the measured probe deceases as $\beta$ get smaller, the unpolarised self-emission intensity seen by the CCD remains constant. Smaller choices of $\beta$ therefore effectively "amplify" the relative intensity of the self-emission with respect to the signal intensity. Greater attenuation is then required to avoid saturating the CCD with this "amplified" self-emission signal, which results in a smaller dynamic range left available for the measurement. Once $\beta$ has been selected, the intensity of the background polarograms is adjusted so they lie at a point in the normalised CCD response range given by the following expression:

$$
N_{B}=\frac{\sin ^{2} \beta}{\sin ^{2}\left(\alpha_{M}+\beta\right)+\frac{I_{S E}}{2 I_{0}}} .
$$

Once $\alpha$ has been measured the next step is to use it to find the magnetic field distribution in the plasma. The equation used to calibrate Faraday rotation measurements is the so called "quasilongitudinal" approximation, ${ }^{18,31}$

$$
\begin{aligned}
\alpha & =\frac{e^{3} \lambda^{2}}{8 \pi^{2} \varepsilon_{0} m_{e}^{2} c^{3}} \int n_{e} \boldsymbol{B} \cdot \boldsymbol{d} z \\
& =2.62 \times 10^{-13} \lambda^{2} \int n_{e} \boldsymbol{B} \cdot \boldsymbol{d} z[S I],
\end{aligned}
$$

where the coordinate system has been selected such that the probe beam propagates along the z-axis. The rotation angle $\alpha$ is thus sensitive only to the component of $\boldsymbol{B}$ lying parallel to the probe propagation vector. The approximation is only valid within certain limits, however for the plasma parameter space accessed in typical Magpie experiments $\left(n_{e} \sim 10^{18} \mathrm{~cm}^{-3}\right.$, $B \sim 1 \mathrm{~T}$, and $\lambda=10^{-6} \mathrm{~m}$ ) it can be shown that it may be 
used safely in almost all cases where the field is not entirely orthogonal to the probe. ${ }^{18,31}$ The equation can be further approximated by replacing the path integral $\int n_{e} \boldsymbol{B} \cdot \boldsymbol{d} z$ with the product of the areal electron density $\int n_{e} d Z$ and average magnetic field parallel to the probe $\overline{\boldsymbol{B}}_{\|}$. Rearrangement leads to an expression for $\overline{\boldsymbol{B}}_{\|}$:

$$
\begin{aligned}
\overline{\boldsymbol{B}}_{\|}(x, y) & =\frac{8 \pi^{2} \varepsilon_{0} m_{e}^{2} c^{3}}{e^{3} \lambda^{2}} \frac{\alpha(x, y)}{\int n_{e}(x, y, z) d z} \\
& =\frac{3.82 \times 10^{12} \alpha(x, y)}{\lambda^{2} \int n_{e}(x, y, z) d z} .
\end{aligned}
$$

A schematic diagram of the overall Faraday rotation diagnostic is shown in Figure 4(a). The linearly polarised probe laser $(1053 \mathrm{~nm}, 1 \mathrm{~ns}, 3 \mathrm{~J}, \varnothing 40 \mathrm{~mm})$ is directed through the plasma, and acquires a spatially varying phase and polarisation rotation due to a combination of the plasma refractive index and the Faraday effect. It passes through a neutral density (ND) filter which attenuates both the probe beam and self-emission by a factor of 100 , and is then optically relayed to an analysis table using a high angular acceptance imaging system. The beam passes through a laser-line filter $(\lambda=1053 \mathrm{~nm}, \delta \lambda \sim 10 \mathrm{~nm})$ which discards the majority of the remaining self-emission and is then split using a nonpolarising cube beam-splitter (NPCBS) to provide one beam for the interferometry channel and one for the polarimetry channel (this split is also shown by Figure 2(a)). The polarimetry beam passes through a second ND filter stack and is then split a second time using a second NPCBS to produce a pair of identical beams for the two polarimetry channels. Each polarimetry beam is analysed using a high extinction ratio $\left(>10^{7}\right)$, high acceptance angle $\left( \pm 20^{\circ}\right)$ polarisation analyser (Thorlabs LPVIS100-MP2). As discussed in the previous paragraphs these are set at angles $\pm \beta$ either side of extinction. Images of the plasma at the midpoint of the plasma object are focused onto a pair of 16 bit CCD cameras (monochrome Atik $383 \mathrm{~L}+$ ). The cameras are left exposed for the duration of the experiment, and therefore the time resolution of the measurement is set by the laser pulse duration $(\sim 1 \mathrm{~ns})$. Background polarograms are captured just before the experiment in order to minimise alignment issues associated with drifting of the optical path. Background image intensities are tuned to optimise the sensitivity of the diagnostic by adjusting the attenuation provided by the second ND filter stack.

An example of the polarimetry data produced by this diagnostic is shown in Figures 4(b)-4(e). This data was captured simultaneously to the interferogram presented in Sec. III (see Figure 2). Magnetic field is embedded in the ablation flow produced by the inverse wire array due to the current driven launch mechanism, and the magnetic Reynolds

(a) Faraday polarimetry layout
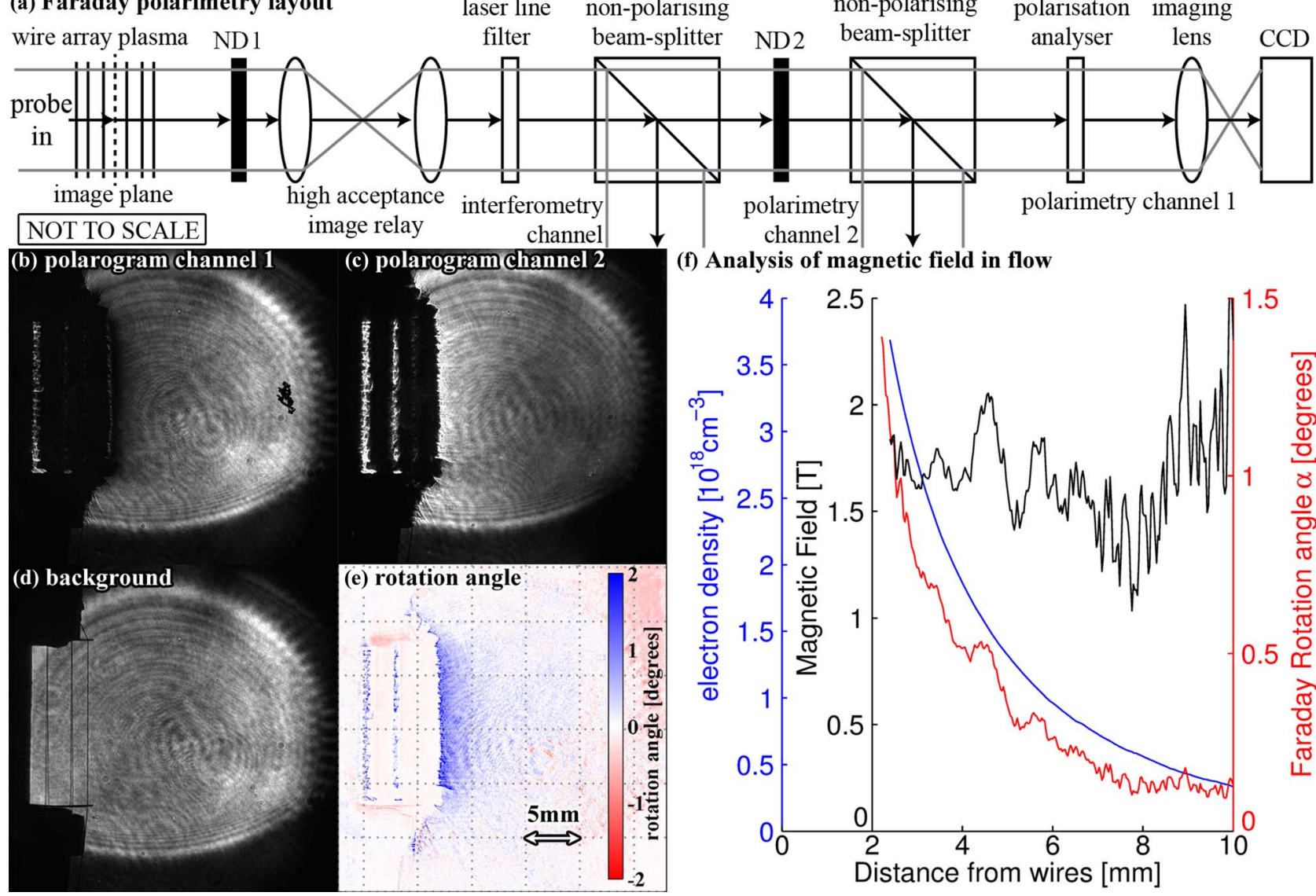

(f) Analysis of magnetic field in flow

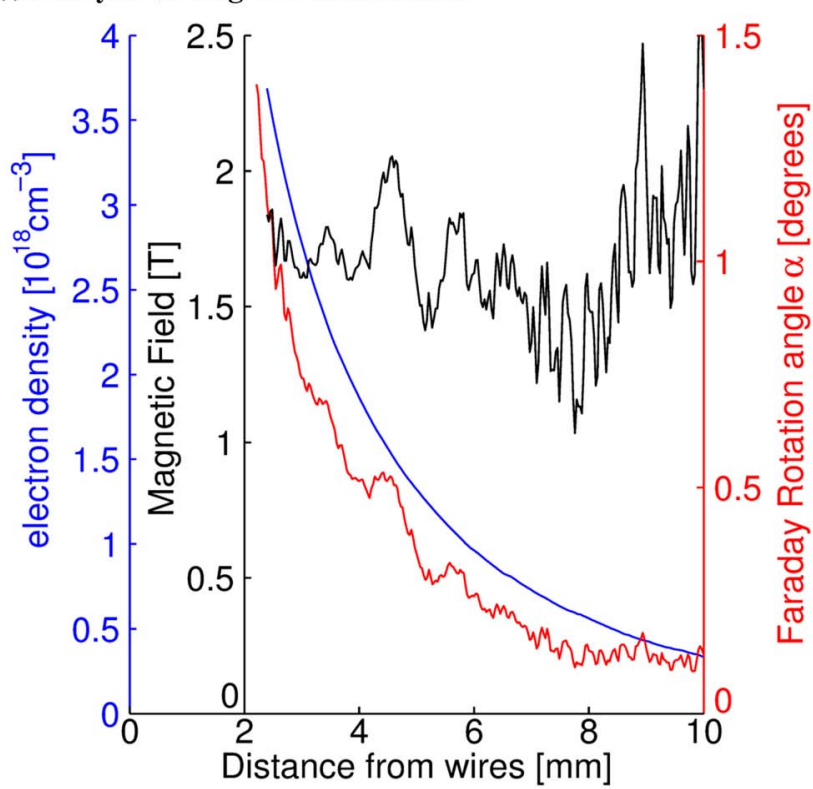

FIG. 4. (a) Schematic of the Faraday rotation diagnostic. (b)-(e) Example of a polarimetry measurement. This data was extracted from the same probe beam as the interferometry data shown in Figure 2. (b) and (c) are the two experimental polarograms, (d) shows an example of a background polarogram, (e) shows a plot of the analysed rotation angle $\alpha$, (f) shows the magnetic field calculated based on $10 \mathrm{~mm}$ height averaged line profiles of $\alpha$ from (e) and the electron density $n_{e}$ from Figure 2(c) in the outflow region. 
number of this plasma is large enough to ensure that a significant fraction of the field is advected with the flow. In these experiments offset angle $\beta$ was set to $4^{\circ}$. Figures 4(b) and 4(c) show the two polarograms captured during the experiment, (d) shows the background polarogram. Darkening of the probe in the outflow region is seen in (b) and brightening in the same region in (c); the extracted rotation angle is plotted in (e), and shows $\alpha \sim 1^{\circ}-2^{\circ}$ in the region of the plasma outflow near the wires. Figure 4(f) shows radial profiles of $\alpha$ from (e), $\int n_{e} d l$ from Figure 2(c) and the corresponding calculated $\overline{\boldsymbol{B}}_{\|}$profile. The line profiles used to produce this plot were calculated by averaging the $\int n_{e} d l$ and $\alpha$ profiles over a height of $10 \mathrm{~mm}$ in the direction parallel to the wires. The overall trends of the electron density and rotation angle appear very similar, and their ratio gives a fairly constant $\overline{\boldsymbol{B}}_{\|}$in the flow of $\sim 2 \pm 0.5 \mathrm{~T}$. The noise in the $\overline{\boldsymbol{B}}_{\|}$plot is caused by the decreasing S/N ratio of the Faraday rotation measurement as the measured rotation decreases. The result that the magnetic field in the plasma remains fairly constant is in itself a novel observation, and merits further investigation.

\section{CONCLUSION}

A suite of laser based diagnostics has been developed in order to study the dynamics of plasma flows produced during pulsed power driven HEDP experiments. Data from experiments investigating ablation flows produced by wire array zpinches have been used to illustrate how measurements from these different diagnostics complement one another and enhance interpretation of the data. In order to properly calibrate Faraday polarimetry measurements in terms of the $\overline{\boldsymbol{B}}_{\|}$in the plasma, interferometric $n_{e}$ measurements are required. These measurements are also used to properly constrain Thomson scattering spectral fits. Recent Thomson scattering measurements of wire array precursors ${ }^{11}$ indirectly indicate the presence of magnetic field on axis; this may be detected directly using the Faraday diagnostic in future experiments. The ability to compare and contrast independent measurements of the various parameters can provide verification of key results and improve interpretation.

\section{ACKNOWLEDGMENTS}

This work was supported in part by EPSRC Grant No. EP/G001324/1, by DOE cooperative Agreement Nos. DEF03-02NA00057 and DE-SC-0001063 and by Sandia National Laboratories. We are pleased to acknowledge joint EPSRC and AWE Aldermaston support for personnel.

\section{APPENDIX: DERIVATION OF SENSITIVITY EQUATION}

The total intensity of any given point in the two Faraday images, $I_{ \pm}$, and maximum expected intensity, $I_{M}$, may be writ- ten in terms of the rotation angle, $\alpha$, the maximum expected value of $\alpha, \alpha_{M}$, the offset angle, $\beta$, the probe intensity $I_{0}$, and the expected self-emission intensity $I_{S E}$ :

$$
I_{ \pm}=I_{0} \sin ^{2}(\alpha \pm \beta)+\frac{I_{S E}}{2} ; \quad I_{M}=I_{0} \sin ^{2}\left(\alpha_{m}+\beta\right)+\frac{I_{S E}}{2} .
$$

The attenuation of the probe beam is adjusted so that the intensity range $\left[0, I_{M}\right]$ covers the entire $C C D$ dynamic range. The figure of merit for sensitivity is then the difference $D$ between the normalised signals measured by each channel:

$$
\begin{aligned}
\frac{I_{ \pm}}{I_{M}} & =\frac{\sin ^{2}(\alpha \pm \beta)+\frac{I_{S E}}{2 I_{0}}}{\sin ^{2}\left(\alpha_{M}+\beta\right)} \\
D & =\frac{I_{+}}{I_{M}}-\frac{I_{-}}{I_{M}}=\frac{\sin (2 \alpha) \sin (2 \beta)}{\left[\sin ^{2}\left(\alpha_{M}+\beta\right)+\frac{I_{S E}}{2 I_{0}}\right]} .
\end{aligned}
$$

Taking the mean of $d D / d \alpha$ over the range $-\alpha_{M} \rightarrow \alpha_{M}$ leads to the expression quoted in the text.

${ }^{1}$ Y. Kuramitsu et al., Phys. Rev. Lett. 106, 175002 (2011).

${ }^{2}$ H.-S. Park et al., High Energy Density Phys. 8, 38 (2012).

${ }^{3}$ J. S. Ross et al., Phys. Plasmas 19, 056501 (2012).

${ }^{4}$ E. C. Merritt et al., Phys. Plasmas 21, 055703 (2014).

${ }^{5}$ I. H. Mitchell et al., Rev. Sci. Instrum. 67, 1533 (1996).

${ }^{6}$ G. F. Swadling et al., Phys. Plasmas 20, 022705 (2013).

${ }^{7}$ S. V. Lebedev et al., Phys. Plasmas 21, 056305 (2014).

${ }^{8}$ G. F. Swadling et al., Phys. Plasmas 20, 062706 (2013).

${ }^{9}$ F. Suzuki-Vidal et al., Phys. Plasmas 19, 022708 (2012).

${ }^{10}$ F. Suzuki-Vidal et al., High Energy Density Phys. 9, 141 (2013).

${ }^{11}$ G. F. Swadling et al., Phys. Rev. Lett. 113, 035003 (2014).

${ }^{12}$ A. J. Harvey-Thompson et al., Phys. Rev. Lett. 108, 145002 (2012).

${ }^{13}$ A. J. Harvey-Thompson et al., Phys. Plasmas 19, 056303 (2012).

${ }^{14}$ F. Suzuki-Vidal et al., IEEE Trans. Plasma Sci. 39, 2830 (2011).

${ }^{15}$ J. Sheffield, D. Froula, S. H. Glenzer, and N. C. Luhmann, Jr., Plasma Scattering of Electromagnetic Radiation: Theory and Measurement Techniques (Academic Press, 2010), p. 104.

${ }^{16}$ E. Hecht, Optics, 3rd ed. (Addison-Wesley, Reading, 1997), pp. 213-214.

${ }^{17} \mathrm{H}$. Zhu and K. Schneider, in Proceedings of the International Conference on 4th GeoComputation, Fredericksbg, VA (Mary Washington College, 1999), pp. 25-28.

${ }^{18}$ I. H. Hutchinson, Principles of Plasma Diagnostics (Cambridge University Press, 2005), pp. 99, 109, and 118.

${ }^{19}$ A. J. Harvey-Thompson et al., Phys. Plasmas 16, 022701 (2009).

${ }^{20}$ G. N. Hall et al., Phys. Plasmas 20, 022703 (2013).

${ }^{21}$ V. V. Aleksandrov et al., Plasma Phys. Rep. 35, 200 (2009).

${ }^{22}$ J. Greenly et al., AIP Conf. Proc. 1088, 53-56 (2009).

${ }^{23}$ F. Suzuki-Vidal et al., Phys. Plasmas 17, 112708 (2010).

${ }^{24}$ P.-A. Gourdain et al., Phys. Plasmas 17, 012706 (2010).

${ }^{25}$ M. Tatarakis et al., Phys. Plasmas 5, 682 (1998).

${ }^{26}$ S. N. Bland et al., Rev. Sci. Instrum. 77, $10 \mathrm{E} 315$ (2006).

${ }^{27}$ V. V. Ivanov et al., IEEE Trans. Plasma Sci. 34, 2247 (2006).

${ }^{28}$ G. Sarkisov, B. Etlicher, and V. Yan'kov, J. Expt. Theor. Phys. 81, 743 (1995).

${ }^{29}$ A. Y. Labetsky et al., IEEE Trans. Plasma Sci. 30, 524 (2002).

${ }^{30}$ N. Qi, S. F. Fulghum, R. R. Prasad, and M. Krishnan, IEEE Trans. Plasma Sci. 26, 1127 (1998).

${ }^{31}$ T. Pisarczyk et al., J. Sov. Laser Res. 11, 1 (1990). 